\title{
Atypical presentation of frosted branch angiitis from Turkey: a case report
}

This article was published in the following Dove Press journal:

Clinical Optometry

16 April 2010

Number of times this article has been viewed

\section{Burak Turgut \\ Mehmet Kaan Kaya \\ Tamer Demir \\ Ulku Celiker \\ Orhan Aydemir}

Department of Ophthalmology, Firat University School of Medicine, Elazig, Turkey
Correspondence: Burak Turgut Department of Ophthalmology, Firat University School of Medicine, Elazig, Turkey

Tel +904242333555

Fax +90 4242388096

Email drburakturgut@gmail.com
Abstract: Frosted branch angiitis (FBA) is a rare kind of retinal angiitis. We report an atypical case of FBA from Turkey. A 35-year-old Turkish woman complained of blurred vision in her left eye. Fundus examination revealed the perivascular white sheathing around major veins, predominantly on the inferior retina, and macular edema in the left eye. A comprehensive search failed to detect any underlying etiology. In systemic examinations, no pathologic finding was obtained except a slight elevation in Epstein-Barr virus (EBV) titer. EBV might be the probable cause of atypical FBA. Thus, the laboratory tests for EBV should also be performed during the etiological examination of patients with FBA.

Keywords: frosted branch angiitis, atypical presentation, Epstein-Barr virus

\section{Introduction}

Frosted branch angiitis (FBA) is a rare kind of retinal angiitis, first reported by Ito and colleagues as idiopathic bilateral retinal periphlebitis. Sixty cases have been reported since 1976, with the majority (75\%) in the literature and most cases have been idiopathic. ${ }^{1,2}$ The condition is typically presented with a bilateral, acute, severe visual acuity loss. Unilateral involvement is rare. To our knowledge and after a literature search through PubMed, only one case has been reported from Turkey. ${ }^{3}$ We report here a case of atypical FBA without severe visual acuity loss and with the predominant involvement of inferior vessels.

\section{Case report}

A 35-year-old Turkish woman presented to the university hospital with the complaint of having blurred vision in her left eye for a period of two months. She had no remarkable history of any systemic and ocular disease. On initial examination, her corrected visual acuity was 20/20 in the right eye and 20/30 in the left eye. The intraocular pressure was $17 \mathrm{mmHg}$ in both eyes. Direct and indirect light reflexes were intact, without a relative afferent pupillary defect. The extraocular movements were full. The anterior segment examination was normal without iris pathology and cellular reaction. Ophthalmoscopy revealed $2+$ cellular reaction in the anterior vitreous. Fundus examination showed perivascular white sheathing on major veins, predominantly in the inferior retina and macular edema in the left eye (Figure 1).

Late phase fluorescein angiograms showed perivenous staining and dye leakage from the vessels with sheathing and macular edema in the same eye (Figure 2). A presumptive diagnosis of acute idiopathic FBA was made and prednisone $(1 \mathrm{mg} / \mathrm{kg} /$ day $)$ treatment 


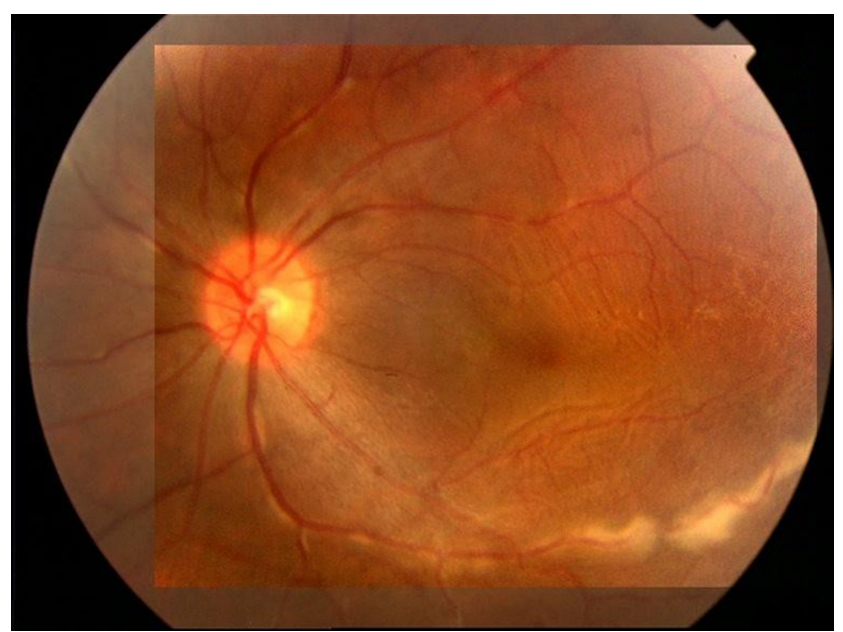

Figure I Color photograph in presentation of the lesion that shows unilateral perivascular white sheathing on major veins, predominantly in the inferior retina.

was initiated. A comprehensive research failed to reveal any underlying etiology.

The tests included: erythrocyte sedimentation rate; plasma protein; urea and angiotensin-1-converting enzyme level; complete blood count; serum immunoglobulins; transaminases and electrolytes; tumor markers; anticardiolipin and antimitochondrial antibodies; and titers for cytomegalovirus (CMV); human immune deficiency virus (HIV); herpes simplex; varicella zoster; toxoplasmosis and syphilis; brucella; and tuberculosis. With these tests, the imaging examinations including, thorax and abdomen computerized tomography's, and cranial magnetic resonance scanning revealed no obvious abnormalities. In systemic examinations, no pathological finding was obtained except a slight elevation in the titers of Epstein-Barr virus (EBV) antibody (EBNA) (31.2 within the first week of the presentation and 479.0

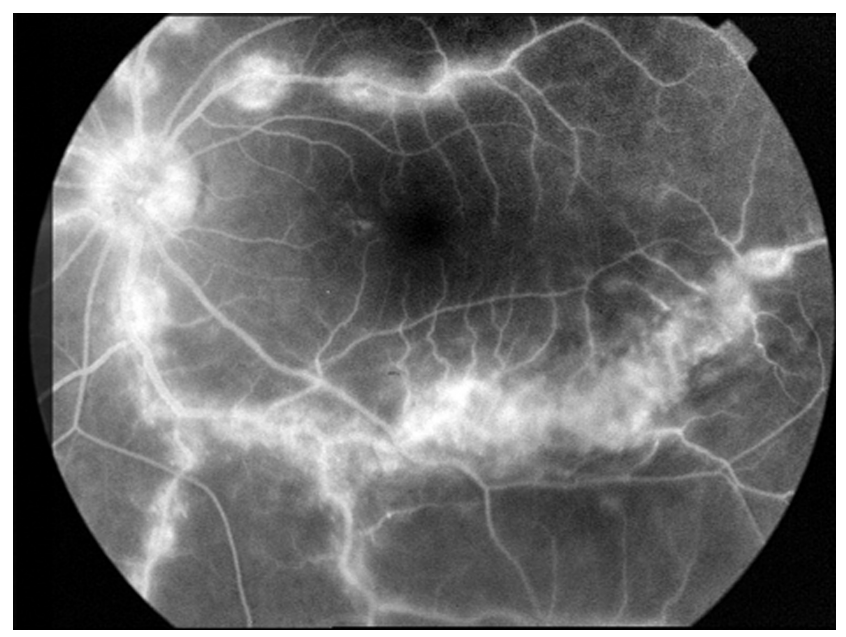

Figure $\mathbf{2}$ Last phase angiogram shows the perivenous staining and dye leakage from the vessels with sheathing and macular edema in the same eye. in the third month of follow-up; normal level being <11). In addition there was also a slight elevation in the titer of EBV-VCA IgG antibody (antibody to EBV-the viral capsid antigen) (23.3 within the first week of presentation and 15.0 in the third month of follow-up; normal level being <11). There was no symptom complex or a history of any systemic disease consistent with EBV infection such as infectious mononucleosis.

Prednisone was tapered over 6 weeks and the patient showed gradual improvement in visual acuity with the regression of perivascular sheathing. At her eight-week follow-up visit, visual acuity was 20/25 in the left eye with macular pucker and mild retinal perivascular sheathing (Figure 3). At the end of the fourth month of follow-up, her visual acuity was identical with the last level, but the patient did not consent to phlebotomy for the antibody titers or to attend any further follow-up visits because of the recovery of her visual acuity.

\section{Discussion}

Acute idiopathic FBA is a clinical entity that predominantly affects the young and healthy. The cause of disease is unknown, and it may be primary (idiopathic) or secondary. However, it has been postulated that it may be due to immune-complex deposition or hypersensitivity reaction to a variety of infectious agents. ${ }^{4,5}$ This supports the use of systemic corticosteroids in the management of the disease. ${ }^{6}$ FBA may be associated with diseases such as lymphoma or leukemia due to the infiltration of malignant cells, infections like: HIV; CMV; toxoplasmosis; or autoimmune diseases such as: sarcoidosis; syphilis; Crohn's disease; or systemic lupus erythematosus. Therefore, these diseases should be considered in the differential diagnosis of FBA. ${ }^{4,5}$ Kleiner proposed that otherwise healthy young patients might be referred to as having "acute idiopathic FBA", because there is the lack of systemic associations. ${ }^{1-5}$ In addition, it has been reported that FBA may accompany: Behçet's disease; multiple sclerosis; rapidly progressive glomerulonephritis; vitreous hemorrhage; and central retinal vein obstruction. ${ }^{2,4-8}$

As in our case, most patients have been treated with systemic corticosteroids with rapid visual recovery within a few weeks. However, there is some data concerning patients who have recovered adequate visual acuity without steroid temic steroids is not well known; and due to the possibility of permanent loss of vision, the use of corticosteroids is recommended. ${ }^{4-6}$ Antiviral drugs for the treatment of FBA have also been used empirically. However, their therapeutic 


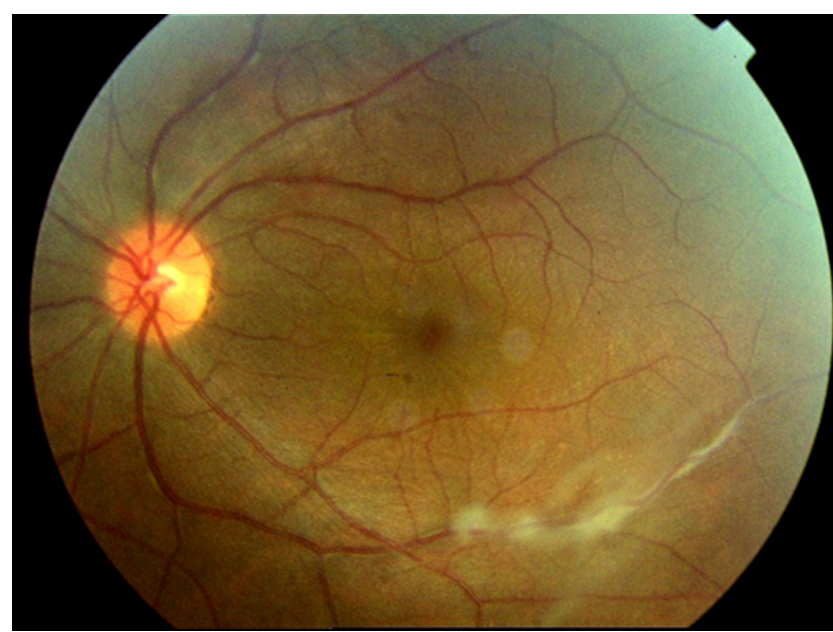

Figure 3 Color photograph following treatment that shows macular pucker and mild retinal perivascular sheathing.

contribution is still unclear. ${ }^{4,5,7}$ Thus, our patient did not receive any antiviral therapy.

In the majority of cases, the typical clinical properties of acute idiopathic FBA were reported to be as follows: no systemic manifestation; acute onset; bilateral involvement; iridocyclitis; and diffuse perivenous sheathing. ${ }^{4,5}$ In the case presented here, the FBA involvement was unilateral, localization was on the major vein arcades and predominantly in the inferior retina, and the patient had no iridocyclitis. Huerva and colleagues reported a case of acute bilateral FBA that showed a positive IgG antibody titer for EBV with the presentation of axillary, submandibular and inguinal lymphadenopathies. ${ }^{9}$ In some ways, our case was similar to that of Huerva and colleagues as the retinal signs appeared to be less severe and the involvement was predominantly in the inferior retina and, although the lymphadenopathy was absent. Recently, Farrando and colleagues reported a case with bilateral FBA associated with systemic EBV infection. ${ }^{10}$ However, their case had the typical clinical manifestations of FBA. The development of FBA in patients with systemic EBV infection may be due to hypersensitivity reaction to viral antigen.

\section{Conclusion}

We presented a rare case of idiopathic unilateral FBA with atypical presentation, because of the predominantly inferior retinal involvement and mild vitritis. To our knowledge, the presented case is the second case reported from Turkey. In view of the detected EBV titer screening for this condition should be considered in patients with FBA, where other serological findings are negative, and in FBA patients which have atypical presentation.

\section{Disclosures}

The authors report no conflicts of interest in this work.

\section{References}

1. Ito Y, Nakano M, Kyu N, Takeuchi M. Frosted branch angiitis in a child. Rinsho Ganka (Jpn J Clin Ophthalmol). 1976;30:797-803.

2. Agrawal S, Agrawal J, Agrawal TP. Frosted branch angiitis. Eye. 2005;19:931-932.

3. Atmaca LS, Gunduz K. Acute frosted retinal periphlebitis. Acta Ophthalmol (Copenh). 1993;71:856-859.

4. Kleiner RC. Frosted branch angiitis. Clinical syndrome or clinical sign? Retina. 1997;17:370-371

5. Walker S, Iguchi A, Jones NP. Frosted branch angiitis: a review. Eye 2004; 18:527-533

6. Hamed LM, Fang EN, Fanous MM, Mames R, Friedman S. Frosted branch angiitis: The role of systemic corticosteroids. $J$ Pediatr Ophthalmol Strabismus. 1992;29:312-313.

7. Narita K, Sato A. Systemic acyclovir was effective in a case of recurrent retinal angiitis. Rinsho Ganka (Jpn J Clin Ophthalmol). 1990;44 739-743

8. Taban M, Sears JE, Crouch E, Schachat AP, Traboulsi EI. Acute idiopathic frosted branch angiitis. JAAPOS. 2007;11:286-287.

9. Huerva V, Puig T, Sanchez MC, Jurjo C, Asenjo J. A new case of acute idiopathic frosted branch angiitis in Europe. Eur J Ophthalmol. 2002;12:127-130.

10. Farrando J, Fonollosa A, Segura A, Garcia-Arumi J. Frosted branch angiitis associated with Epstein-Barr virus systemic infection. Ocul Immunol Inflamm. 2008;16:41-43.

\section{Publish your work in this journal}

Clinical Optometry is an international, peer-reviewed, open access journa publishing original research, basic science, clinical and epidemiological studies, reviews and evaluations on clinical optometry. All aspects of patient care are addressed within the journal as well as the practice of optometry including economic and business analyses. Basic and clinical

\section{Dovepress}

research papers are published that cover all aspects of optics, refraction and its application to the theory and practice of optometry. The manuscript management system is completely online and includes a very quick and fair peer-review system, which is all easy to use. Visit http://www.dovepress. com/testimonials.php to read real quotes from published authors. 